

Stroke \& Vascular Neurology

\title{
Tenecteplase Reperfusion therapy in Acute ischaemic Cerebrovascular Events-II (TRACE II): rationale and design
}

\author{
Shuya Li (D) ,1,2 Bruce C V Campbell, ${ }^{3}$ Lee H Schwamm, ${ }^{4}$ Marc Fisher, ${ }^{5}$ \\ Mark Parsons, ${ }^{6} \mathrm{Hao}$ Li (D) , ${ }^{1}$ Yuesong Pan (D) , ${ }^{1}$ Yongjun Wang, ${ }^{1,2}$ On behalf of the \\ TRACE II investigators
}

To cite: Li S, Campbell BCV, Schwamm LH, et al. Tenecteplase Reperfusion therapy in Acute ischaemic Cerebrovascular Events-II (TRACE II): rationale and design. Stroke \& Vascular Neurology 2022;7: 001074. doi:10.1136/ svn-2021-001074

- Additional supplemental material is published online only. To view, please visit the journal online (http://dx.doi.org/10. 1136/svn-2021-001074).

Received 23 April 2021 Accepted 2 August 2021 Published Online First 26 August 2021

\section{Check for updates}

(c) Author(s) (or their employer(s)) 2022. Re-use permitted under CC BY-NC. No commercial re-use. See rights and permissions. Published by BMJ.

For numbered affiliations see end of article.

Correspondence to Dr Yongjun Wang; yongjunwang@ncrcnd.org.cn

\section{ABSTRACT}

Background and purpose Tenecteplase (TNK) is a promising agent for treatment of acute ischaemic stroke (AIS). We hypothesised that recombinant human TNK tissue-type plasminogen activator (rhTNK-tPA) is noninferior to rt-PA in achieving excellent functional outcome at 90 days, when administered within 4.5 hours of ischaemic stroke onset.

Methods and design Tenecteplase Reperfusion therapy in Acute ischemic Cerebrovascular Events (TRACE) is a phase III, multicentre, prospective, randomised, open-label, blinded-end point non-inferiority study. Patients eligible for intravenous thrombolysis therapy are randomised to rhTNK-tPA $0.25 \mathrm{mg} / \mathrm{kg}$ (single bolus) to a maximum of $25 \mathrm{mg}$ or rt-PA $0.9 \mathrm{mg} / \mathrm{kg}$ ( $10 \%$ bolus $+90 \%$ infusion $/ 1$ hour) to a maximum of $90 \mathrm{mg}$. Medications considered necessary for the patient's health may be given at the discretion of the investigator during 90-day follow-up. Study outcomes The primary study outcome is excellent functional outcome defined as modified Rankin Scale (mRS) $0-1$ at 90 days. Secondary efficacy outcomes include favourable functional outcome defined as $\mathrm{mRS} \leq 2$ at 90 days, ordinal distribution of $\mathrm{mRS}$ and major neurological improvement on the National Institutes of Health Stroke Scale. Safety outcomes are symptomatic intracranial haemorrhage within 36 hours and death from any cause.

Discussion There is no completed registration study of TNK in AIS worldwide. TRACE II strives to provide evidence for a new drug application for rhTNK-tPA in AIS within 4.5 hours through a well-designed and rigorously executed randomised trial in China.

Trial registration number NCT04797013.

\section{INTRODUCTION}

The burden of stroke continues to increase worldwide. In China, stroke has become the leading cause of death, with ischaemic stroke being the dominant type. ${ }^{1-3}$ Intravenous thrombolysis is of proven clinical benefit for eligible patients with acute ischaemic stroke (AIS) and recombinant tissue plasminogen activator (rtPA) is the only licensed thrombolytic agent within 4.5 hours after symptom onset. ${ }^{4-6}$
Tenecteplase (TNK) is a genetically engineered modified form of rt-PA with practical delivery advantages and is currently approved to treat acute myocardial infarction (AMI) in many countries. ${ }^{7}$ In China, Metalyse and recombinant human TNK tissue-type plasminogen activator for injection (rhTNK-tPA) are approved for AMI indication. ${ }^{8}$ There is no completed registration study of TNK in AIS worldwide. Several phase II and III studies of TNK initiated by investigators provided information on its safety and efficacy for the treatment of AIS. ${ }^{10-14}$ A meta-analysis of 5 randomised studies, including 1585 patients in total (828 TNK, 757 rt-PA), showed that the modified Rankin Scale (mRS) 0-1 at 90 days was $57.9 \%$ in TNK-treated patients vs $55.4 \%$ in rt-PA-treated patients with a risk difference of $4 \%(95 \% \mathrm{CI}-1 \%$ to $8 \%)$. For safety end points, TNK and rt-PA demonstrated a risk difference of $0 \%$ in symptomatic intracranial haemorrhage (sICH, $95 \% \mathrm{CI}-1 \%$ to $2 \%$ ) and 90-day mortality $(95 \% \mathrm{CI}-3 \%$ to $2 \%) .^{15}$

Although there is accumulating evidence supporting the use of TNK in patients with AIS, these data came from trials conducted in Caucasians. The optimal dosage of rhTNK-tPA for Chinese patients with AIS has not yet been demonstrated. A dose of $0.25 \mathrm{mg} / \mathrm{kg}$ may be suggested for future efficacy studies in Caucasians patients with AIS based on the results of contemporary clinical studies. ${ }^{4} 12$ A phase II study of rhTNK-tPA in China showed that rhTNK-tPA was well tolerated in Chinese patients with AIS similar to the Caucasians at dosages of $0.25 \mathrm{mg} / \mathrm{kg}$ administered within 3 hours of symptom onset (results to be published). Tenecteplase Reperfusion therapy in Acute ischemic Cerebrovascular Events-II (TRACE II), a phase III study, aiming to demonstrate that rhTNK-tPA $0.25 \mathrm{mg} / \mathrm{kg}$ is non-inferior to rt-PA $0.9 \mathrm{mg} / \mathrm{kg}$ for intravenous 
thrombolysis of patients with AIS in China. The efficacy and safety results of this study may support a new drug application for rhTNK-tPA in AIS within 4.5 hours.

\section{METHODS}

\section{Study organisation and design}

The steering committee of TRACE II is the highest decision-making body and express opinions freely and independently at scheduled or unscheduled meetings (online supplemental file 1). It is responsible for supervising the study executive. An independent data monitoring committee will maintain surveillance of participant safety and advise the steering committee about any safety concerns (table 1 ).

TRACE II is a phase III, multicentre, prospective, randomised, open-label, blinded-end point (PROBE) non-inferiority study. ${ }^{16}$ Randomisation will be $1: 1$ for intravenous thrombolysis with rhTNK-tPA versus rt-PA via a centralised website. Investigational product should be administrated no later than 4.5 hours after symptom onset. Patients will be followed up 90 days to assess the primary outcome. Approximately 70 stroke centres in

Table 1 Trial assessment flow chart

\begin{tabular}{|c|c|c|c|c|c|c|}
\hline \multirow[b]{2}{*}{ Procedure/Investigation } & \multirow{2}{*}{$\begin{array}{l}\text { Baseline } \\
-4.5 \text { hours }\end{array}$} & \multirow{2}{*}{$\begin{array}{l}\text { Treatment } \\
0 \text { hour }\end{array}$} & \multicolumn{4}{|l|}{ Follow-up } \\
\hline & & & $24 \pm 2$ hours & 24-36 hours & Day $7 \pm 1$ or discharge & Day $90 \pm 7$ \\
\hline Informed consent ${ }^{*}$ & $x$ & & & & & \\
\hline Symptoms of the disease & $x$ & & & & & \\
\hline Medical history $\dagger$ & $x$ & & & & & \\
\hline Vital signs§ & $x$ & $x$ & $x$ & & $x$ & \\
\hline mRSף & $x \S$ & & & & & $x$ \\
\hline NIHSS & $x$ & & $x$ & & $x$ & \\
\hline Barthel Index & & & & & & $\mathrm{X}$ \\
\hline Haematology & $x$ & & $\mathrm{x}$ & & $x$ & \\
\hline Clinical chemistry & $x$ & & $x$ & & $x$ & \\
\hline Coagulation profile & $x$ & & $x$ & & $x$ & \\
\hline Urinalysis & & & $x$ & & $\mathrm{X}$ & \\
\hline 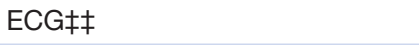 & $x$ & & & & $\mathrm{x}$ & \\
\hline Imaging§§ & $x$ & & & $x$ & & \\
\hline Randomisation & $x$ & & & & & \\
\hline Thrombolysis information $\ddagger$ & & $x$ & & & & \\
\hline $\begin{array}{l}\text { Adverse events/Serious adverse } \\
\text { events assessment }\end{array}$ & & $\mathrm{X}$ & $\mathrm{X}$ & $\mathrm{X}$ & $x$ & $\mathrm{X}$ \\
\hline
\end{tabular}

*Patients who had symptoms on awakening or unknown onset were excluded in the study.

†Prior medication is recorded only for those drugs that need to be restricted in the eligibility criteria.

$\ddagger$ Thrombolysis information includes the time of thrombolytic therapy in both groups (including the intravenous bolus time, the starting and ending time of maintenance infusion, the dose of the bolus and the maintenance infusion, the adverse events); pay attention to collecting information of bridging treatment.

$\S$ The baseline blood pressure test is collected at the time of vital signs collection; The ' 0 hour' visit is regarded as within 5 min before thrombolysis; vital signs include blood pressure, pulse, temperature and breath.

IThe mRS scores screened into the group indicate preonset score.

**Pregnancy test is limited to female subjects of childbearing age.

††Laboratory assessments: (1) baseline laboratory assessments include haematology, clinical chemistry and coagulation profile; no need to repeat the assessments performed after the attack and before thrombolysis. Fasting blood is required for the baseline lipid profile (total cholesterol, low-density lipoprotein, high-density lipoprotein, triglycerides). Fast glucose is allowed to decide eligibility criteria with serum glucose collected synchronously. The clinical chemistry results are available after the administration of study drugs. The investigators will deal with the abnormal results according to guidelines and the clinical pathway if necessary. (2) The laboratory assessments of 72 hours after thrombolysis, including haematology, clinical chemistry, coagulation profile and urinalysis, can be acceptable for the 24 hours visit. (3) Haematology, clinical chemistry, coagulation profile and urinalysis should be done at $7 \pm 1$ days or before discharge (whichever occurs first).

捛o need to repeat ECG after the attack and before thrombolysis.

$\S \S$ The baseline imaging, whatever 'CT or MRI', is used to exclude intracranial haemorrhage. Imaging data from another hospital is accepted according to investigators. The follow-up imaging (CT or MRI) should be completed within 24-36 hours to detect intracranial haemorrhage. mRS, modified Rankin Scale; NIHSS, National Institutes of Health Stroke Scale. 
China will participate. Estimated study duration is 32 months.

\section{Patient population}

Inclusion criteria ${ }^{417}$

1. Age is $\geq 18$ years.

2. AIS symptom onset $\leq 4.5$ hours, onset time refers to the time the patient was last known to be well.

3. Prestroke mRS score $\leq 1$.

4. Baseline National Institutes of Health Stroke Scale (NIHSS) 5-25 (inclusive).

5. Informed consent from the patient or legally authorised representative.

\section{Exclusion criteria}

1. Intention to proceed to endovascular treatment.

2. Allergy to TNK or alteplase.

3. Rapidly improving symptoms at the discretion of the investigator.

4. NIHSS consciousness score (NIHSS 1a) $>2$.

5. Arterial puncture at a non-compressible site within the previous 7 days, major surgery within the previous 14 days, sever trauma, gastrointestinal or urinary tract haemorrhage within the previous 21 days.

6. Myocardial infarction or ischaemic stroke in previous 3 months, previous ICH (including parenchymal haemorrhage, intraventricular haemorrhage, subarachnoid haemorrhage, subdural/external haematoma, etc), severe brain trauma, intracranial or intraspinal surgery in previous 3 months or known malignant intracranial neoplasm, giant intracranial aneurysm or arteriovenous malformation.

7. Persistent blood pressure elevation (systolic $\geq 180 \mathrm{~mm}$ $\mathrm{Hg}$ or diastolic $\geq 100 \mathrm{~mm} \mathrm{Hg}$ ), despite blood pressurelowering treatment.

8. Blood glucose $<2.8 \mathrm{mmol} / \mathrm{L}$ or $>22.22 \mathrm{mmol} / \mathrm{L}$ (point-of-care glucose testing is acceptable).

9. Any known defect in coagulation, for example, current use of oral warfarin anticoagulant with an international normalised ratio $>1.7$ or prothrombin time $>15 \mathrm{~s}$, or heparins during the last 48 hours, or use of direct thrombin inhibitors or direct factor Xa inhibitors during the last 48 hours or with an elevated activated partial thromboplastin time greater than the upper limit of normal.

10. Known defect of platelet or clotting function, platelet count below $100 \times 10^{9} / \mathrm{L}$ (note that patients on antiplatelet agents can be included).

11. Weakness after witnessed or presumed seizure that cannot be explained by acute ischaemic injury on brain imaging.

12. Hypodensity in $>1 / 3$ middle cerebral artery territory on non-contrast computer tomography (NCCT).

13. ICH, subarachnoid haemorrhage or other brain haemorrhage identified by CT or MRI.

14. Any terminal illness such that patient would not be expected to survive $>1$ year.

15. Pregnant women, nursing mothers.
16. Inability to adhere to the trial protocol or follow-up.

17. Participation in another clinical trial within the previous 3 months.

18. Any condition that, in the judgement of the investigator could impose hazards to the patient if study therapy is initiated or affect the participation of the patient in the study.

\section{Baseline measures}

Patients who are clinically suspected of AIS within 4.5 hours after symptom onset will be assessed for intravenous thrombolysis eligibility after CT scan. Demographics, medical history, current medications and baseline laboratory tests will be collected. Baseline neurological impairment (eg, NIHSS) and prestroke functional (eg, mRS) scores before this attack will be performed by a certified physician with uniform and standardised training. Primary or comprehensive stroke centres will be selected as study sites to make sure that patients can be assessed and treated with rapid workflow.

\section{Randomisation and blinding}

The proportion of patients in the rhTNK-tPA group and the rt-PA group is 1:1 in each study site. Central web-based randomisation system (Randomization and Trial Supply Management V.3.1.2, Beijing Bioknow Information Technology, China) will be used for randomisation in dynamic blocks of four to balance distribution of group assignments at any time. The size of the blocks will be withheld from the investigators to make sure that they are unaware of the treatment assignments. The intravenous thrombolytic treatment is open label. Investigators who involved in the subsequent clinical and imaging assessment of outcomes are blinded to treatment allocation.

\section{Treatment intervention}

Intervention group: rhTNK-tPA (0.25 mg/kg, max $25 \mathrm{mg})$

rhTNK-tPA $(0.25 \mathrm{mg} / \mathrm{kg})$ is given as a single, intravenous bolus (over 5-10 s) immediately on randomisation. Maximum dose is $25 \mathrm{mg}$.

\section{Control group: rt-PA $(0.9 \mathrm{mg} / \mathrm{kg}$, $\max 90 \mathrm{mg})$}

Ten per cent dose of rt-PA $(0.9 \mathrm{mg} / \mathrm{kg})$ is given as bolus and the remainder over 1 hour infusion. Maximum dose is $90 \mathrm{mg}$.

Patients for whom endovascular treatment is planned are excluded from this study. Patients who are subsequently judged to require endovascular treatment after intravenous thrombolysis in the judgement of the investigator will be included in the intention-to-treat analysis, but will be excluded from per-protocol analysis in order to avoid the effect on the outcome.

\section{Outcomes and follow-up}

Study visits will be performed on the day of randomisation, at 24 hours and 24-36 hours after randomisation, at day 7 and at day 90. On admission, CT with or without CT angiography (CTA) (or MRI/MR angiography (MRA)) is performed. At 24-36 hours after randomisation, CT, 
CTA with CT perfusion or MRA with diffusion-weighted magnetic resonance imaging (DW-MRI) is performed. At randomisation and during follow-up visits, including but not limited to clinical information will be collected: neurological evaluation (mRS and NIHSS); a physical examination, vital signs, concomitant medications and adverse events (AEs). Information is collected at a faceto-face consultation, mRS allows telephone interview (table 1).

Serious adverse events (SAEs) will be reported by investigators adhering to the protocol and Good Clinical Practice guidelines. Two members of the independent Clinical Events Committee (CEC) will adjudicate the report. SAEs and AEs will be tabulated using standard terminology.

\section{Primary outcome}

Proportion of patients with excellent functional outcome defined as $\mathrm{mRS}$ score $\leq 1$ point at 90 days.

\section{Secondary outcomes}

\section{Efficacy outcomes}

1. Proportion of participants with $\mathrm{mRS} \leq 2$ at 90 days.

2. Ordinal distribution of $\mathrm{mRS}$ at 90 days.

3. Proportion of participants with improvement on NIHSS of $\geq 4$ points or a score $\leq 1$ at 24 hours and at 7 days or discharge (whichever occurs first).

4. Barthel Index score $\geq 95$ at day 90 .

\section{Safety outcomes}

1. sICH within 36 hours (as defined by Safe Implementation of Thrombolysis in Stroke ${ }^{18}$ and The European Cooperative Acute Stroke Study III ${ }^{19}$ ).

2. Systemic bleeding at 90 days (as defined by The Global Utilization of Streptokinase and Tissue Plasminogen Activator for Occluded Coronary Arteries ${ }^{20}$ ).

3. Death from any cause within 90 days.

4. AEs/SAEs within 90 days.

\section{Clinical Events Committee}

The CEC consists of experienced neurologists and cardiologists who are not involved in the execution of the study. Membership of the CEC (including qualifications, roles and responsibilities) shall be confirmed by the steering committee. Clinical end point events include: new vascular events, sICH, systematic bleeding and death. All clinical end points are first determined by the investigator, then verified and adjudicated by the CEC based on clinical symptoms, laboratory tests and imaging data.

\section{Data monitoring committee}

An independent data monitoring committee (DMC) will protect the interests of the participants during the study. The DMC need to review the safety and efficacy data gathered during the study, especially serious unexpected adverse reactions, evaluate the risks and benefits regularly and dynamically, provide professional advice to the steering committee based on the analysis results carried out by an independent statistician. A DMC Charter details roles, composition, responsibilities and procedures to ensure maintenance of independence and objectivity of the DMC (online supplemental file 2).

\section{Sample size and statistical analysis}

Sample size

TRACE II uses a non-inferiority design with the primary efficacy outcome being the proportion of patients achieving mRS $0-1$ at 90 days. Based on meta-analysis of previous trials, the risk ratio for the effect of rt-PA versus placebo for the outcome of mRS $0-1$ was 1.24 (95\% CI 1.14 to 1.36$).{ }^{21}$ Prior data indicate that TNK is unlikely to be less safe or effective than alteplase and is more convenient. Under these circumstances, the Food and Drug Administration guidelines for non-inferiority design allow for a relaxation of non-inferiority criteria to preserve at least $50 \%$ of the effect of alteplase. In the TRACE trial, TNK will therefore be declared non-inferior if the lower $97.5 \%$ one-sided CI of the risk ratio for the primary outcome does not cross 0.937 . The phase II TRACE study found that $59.3 \%$ of rt-PA-treated patients achieved mRS $0-1$ at 90 days. The risk ratio boundary of 0.937 would therefore correspond to a $5.9 \%$ absolute risk difference between the rhTNK-tPA group and the rt-PA group. This $3.74 \%$ risk difference margin is regarded as acceptable by clinical experts. Assuming a power of $85 \%$, a one-sided alpha level of 0.025 and an absolute relative risk of 1.07 (63.6\% in rhTNK-tPA vs 59.3\% in rt-PA group with mRS $0-1$ at 90 days based on the TRACE data), the sample size for each group is 614 patients. Allowing for a loss-to-follow-up rate of $10 \%$, the final sample size estimate is 1364 patients (682 in each treatment group).

\section{Statistical analysis}

The primary efficacy analysis will be on an intention-totreat basis and compared using two-sided significance tests. Fisher's exact probability method or $\chi^{2}$ test will be used for comparison of categorical variables, Wilcoxon rank-sum test for comparison of ordinal variables, $\mathrm{T}$ test or rank-sum test for comparison of continuous variables and mean difference of score with $95 \%$ CI will be calculated. The Cochran-Mantel-Haenszel $\chi^{2}$ test considering the central effect will be used for comparison of primary end points between groups, and the $95 \%$ CI of OR was calculated. The odds of achieving mRS $0-1$ at 90 days will be analysed using logistic regression with adjustment of baseline NIHSS and age. OR and the 95\% CI will be reported. Per-protocol analysis will be performed according to whether bridging therapy was assigned. No interim analysis is planned in this trial. All statistical analyses will be performed using SAS V.9.4 software.

\section{DISCUSSION}

As a major public health problem in the world, improving treatment of AIS deserves continuous effort. An improved intravenous thrombolytic approach could have a major impact on health delivery. TNK is a genetically engineered modified form of rt-PA with better fibrin specificity, high resistance to plasminogen-activator inhibitor-1 and slower 
plasma clearance permitting bolus administration which may facilitate efficient institution of reperfusion therapy.

The TRACE II study excludes patients intended to have endovascular thrombectomy. Although several studies of direct endovascular thrombectomy have reported similar results with and without bridging alteplase in the subgroup of patients who present directly to an endovascular-capable centre, there is residual uncertainty about the best treatment approach and intravenous thrombolysis remains the recommended treatment for patients with AIS within 4.5 hours. ${ }^{22-25}$ Previous studies reported that patients with AIS with large vessel occlusion (LVO) receiving intravenous thrombolysis with TNK having favourable clinical outcomes at 3 months compared with patients receiving intravenous alteplase. ${ }^{26} 27$ Subgroup analysis of the patients with AIS with LVO will be conducted in TRACE II study. A separate TRACE III trial will focus to assess the effect of TNK for patients with AIS with LVO in the late time window (4.5-24 hours after stroke onset).

A double-blind design is considered optimal for phase III randomised controlled trials. However, a double-blind and double-dummy drug administration would significantly increase the complexity of the trial procedure in the very narrow time window and delaying drug administration for patients with AIS is unethical. A PROBE design was therefore chosen for TRACE II. Although some investigators in the local centres may be aware of the treatment allocation, investigators who are involved in the subsequent clinical and imaging assessment of outcomes are blinded to treatment allocation.

The most common AEs in clinical studies of thrombolytic agents are haemorrhage, including ICH and systemic bleeding. The safety profile of TNK appears similar to alteplase and TRACE II uses standard thrombolytic therapy exclusions to reduce the risk of bleeding. Angiooedema can occur with both TNK and alteplase and is managed according to standard treatment guidelines. No patients developed angio-oedema in the TRACE phase II study.

In conclusion, TRACE II addresses a critical clinical question in patients with ischaemic stroke. Demonstration of non-inferiority of TNK in this trial would support implementation of convenient intravenous thrombolysis with TNK for patients with AIS.

\section{Author affiliations \\ ${ }^{1}$ China National Clinical Research Center for Neurological Diseases, Beijing Tiantan Hospital, Capital Medical University, Beijing, China \\ ${ }^{2}$ Department of Neurology, Beijing Tiantan Hospital, Capital Medical University, Beijing, China \\ ${ }^{3}$ Department of Medicine and Neurology, University of Melbourne, Melbourne, Victoria, Australia \\ ${ }^{4}$ Department of Neurology and Comprehensive Stroke Center, Massachusetts General Hospital, Harvard Medical School, Boston, Massachusetts, USA ${ }^{5}$ Neurology, Beth Israel Deaconess Medical Center, Boston, Massachusetts, USA ${ }^{6}$ Department of Neurology, Liverpool Hospital, South Western Sydney Clinical School, Sydney, New South Wales, Australia}

Correction notice The article has been corrected since published onlinefirst. The value has been corrected from " $59.4 \%$ " to " $59.3 \%$ " in the sentence "The phase II
TRACE study found..." Also, the phrase " $59.3 \%$ in rhTNK-tPA vs $63.6 \%$ " has been corrected to "63.6\% in rhTNK-tPA vs $59.3 \%$ " in the sentence "Assuming a power of $85 \%$, a one-sided ...".

Acknowledgements The authors extend their special thanks to the physicians as well as all other personnel who dedicated themselves to the TRACE II study. The Steering Committee: Yongjun Wang, Bruce CV Campbell (AUS), Lee H Schwamm (USA), Marc Fisher (USA), Mark W Parsons (AUS). Data Monitoring Committee: Hao Li, David Wang (USA), Lawrence Wong (HK), Dongsheng Fan, Magdy Selim (USA). Independent statistician: Yuesong Pan. Clinical Events Committee: Wenli Hu, Yonghua Huang, Lv Qiang.

Contributors Obtained funding, concept and design: YW. All authors contributed to the study design. Drafting of the manuscript: SL. Critical revision of the manuscript for important intellectual content: BCVC, LHS. Statistical analysis: HL, YP. All authors critically reviewed the manuscript and approved the submitted version.

Funding This study is supported by the National Natural Science Foundation of China (81870905), the National Key R\&D Program of China (2017YFC1308204) and the National Science and Technology Major Project (2017ZX09304018). This study was sponsored and funded by Guangzhou Recomgen Biotech Co., Ltd.

Competing interests None declared.

Patient consent for publication Not required.

Ethics approval All procedures performed in studies involving human participants were in accordance with the ethical standards of the institutional research committee and the principles of the Declaration of Helsinki. All participants gave informed consent before taking part. This study obtained Ethics approval of Institutional Review Board of Beijing Tiantan Hospital, Capital Medical University with number YW2020-046-04.

Provenance and peer review Not commissioned; externally peer reviewed. Data availability statement Data are available on reasonable request. None.

Open access This is an open access article distributed in accordance with the Creative Commons Attribution Non Commercial (CC BY-NC 4.0) license, which permits others to distribute, remix, adapt, build upon this work non-commercially, and license their derivative works on different terms, provided the original work is properly cited, appropriate credit is given, any changes made indicated, and the use is non-commercial. See: http://creativecommons.org/licenses/by-nc/4.0/.

\section{ORCID iDs}

Shuya Li http://orcid.org/0000-0002-7263-0365

Hao Li http://orcid.org/0000-0002-8591-4105

Yuesong Pan http://orcid.org/0000-0003-3082-6789

\section{REFERENCES}

1 GBD 2016 Lifetime Risk of Stroke Collaborators, Feigin VL, Nguyen $\mathrm{G}$, et al. Global, regional, and Country-Specific lifetime risks of stroke, 1990 and 2016. N Engl J Med 2018;379:2429-37.

2 Zhou M, Wang H, Zeng X, et al. Mortality, morbidity, and risk factors in China and its provinces, 1990-2017: a systematic analysis for the global burden of disease study 2017. The Lancet 2019;394:1145-58.

3 Wang Y-J, Li Z-X, Gu H-Q, et al. China stroke statistics 2019: a report from the National center for healthcare quality management in neurological diseases, China national clinical research center for neurological diseases, the Chinese stroke association, National center for chronic and non-communicable disease control and prevention, Chinese center for disease control and prevention and Institute for global neuroscience and stroke collaborations. Stroke Vasc Neurol 2020;5:211-39.

4 Powers WJ, Rabinstein AA, Ackerson T, et al. Guidelines for the early management of patients with acute ischemic stroke: 2019 update to the 2018 guidelines for the early management of acute ischemic stroke: a guideline for healthcare professionals from the American heart Association/American stroke association. Stroke 2019;50:e344-418.

5 Emberson J, Lees KR, Lyden P, et al. Effect of treatment delay, age, and stroke severity on the effects of intravenous thrombolysis with alteplase for acute ischaemic stroke: a meta-analysis of individual patient data from randomised trials. Lancet 2014;384:1929-35.

6 Liu L, Chen W, Zhou H, et al. Chinese stroke association guidelines for clinical management of cerebrovascular disorders: Executive summary and 2019 update of clinical management of ischaemic cerebrovascular diseases. Stroke Vasc Neurol 2020;5:159-76.

7 Llevadot J, Giugliano RP, Antman EM. Bolus fibrinolytic therapy in acute myocardial infarction. JAMA 2001;286:442-9. 
8 Assessment of the Safety and Efficacy of a New Thrombolytic (ASSENT-2) Investigators, Van De Werf F, Adgey J, et al. Singlebolus tenecteplase compared with front-loaded alteplase in acute myocardial infarction: the ASSENT-2 double-blind randomised trial. Lancet 1999;354:716-22.

9 Wallentin L, Goldstein P, Armstrong PW, et al. Efficacy and safety of tenecteplase in combination with the low-molecular-weight heparin enoxaparin or unfractionated heparin in the prehospital setting: the Assessment of the Safety and Efficacy of a New Thrombolytic Regimen (ASSENT)-3 PLUS randomized trial in acute myocardial infarction. Circulation 2003;108:135-42.

10 Parsons M, Spratt N, Bivard A, et al. A randomized trial of tenecteplase versus alteplase for acute ischemic stroke. $N$ Engl J Med 2012;366:1099-107.

11 Huang X, Cheripelli BK, Lloyd SM, et al. Alteplase versus tenecteplase for thrombolysis after ischaemic stroke (ATTEST): a phase 2, randomised, open-label, blinded endpoint study. Lancet Neurol 2015;14:368-76.

12 Campbell BC, Mitchell PJ, Churilov L, et al. Tenecteplase versus alteplase before endovascular thrombectomy (EXTEND-IA TNK): a multicenter, randomized, controlled study. Int J Stroke 2018;13:328-34.

13 Campbell BCV, Mitchell PJ, Churilov L, et al. Effect of intravenous tenecteplase dose on cerebral reperfusion before thrombectomy in patients with large vessel occlusion ischemic stroke: the EXTEND-IA TNK Part 2 randomized clinical trial. JAMA 2020;323:1257-65.

14 Logallo N, Novotny V, Assmus J, et al. Tenecteplase versus alteplase for management of acute ischaemic stroke (NOR-TEST): a phase 3 , randomised, open-label, blinded endpoint trial. Lancet Neurol 2017;16:781-8.

15 Burgos AM, Saver JL. Evidence that tenecteplase is Noninferior to alteplase for acute ischemic stroke: meta-analysis of 5 randomized trials. Stroke 2019;50:2156-62.

16 Hansson L, Hedner T, Dahlöf B. Prospective randomized open blinded end-point (probe) study. A novel design for intervention trials. Blood Press 1992;1:113-9.

17 Berge E, Whiteley W, Audebert H, et al. European stroke organisation (ESO) guidelines on intravenous thrombolysis for acute ischaemic stroke. Eur Stroke J 2021;6:I-LXII.
18 Wahlgren N, Ahmed N, Dávalos A, et al. Thrombolysis with alteplase for acute ischaemic stroke in the safe implementation of thrombolysis in Stroke-Monitoring study (SITS-MOST): an observational study. Lancet 2007;369:275-82.

19 Hacke W, Kaste M, Bluhmki E, et al. Thrombolysis with alteplase 3 to 4.5 hours after acute ischemic stroke. $N$ Engl J Med 2008;359:1317-29.

20 Berkowitz SD, Granger CB, Pieper KS, et al. Incidence and predictors of bleeding after contemporary thrombolytic therapy for myocardial infarction. the global utilization of streptokinase and tissue plasminogen activator for Occluded coronary arteries (GUSTO) I Investigators. Circulation 1997;95:2508-16.

21 Emberson J, Lees KR, Lyden P, et al. Effect of treatment delay, age, and stroke severity on the effects of intravenous thrombolysis with alteplase for acute ischaemic stroke: a meta-analysis of individual patient data from randomised trials. Lancet 2014;384:1929-35

22 Yang $P$, Zhang Y, Zhang L, et al. Endovascular thrombectomy with or without intravenous alteplase in acute stroke. $N$ Engl J Med 2020;382:1981-93.

23 Nogueira RG, Tsivgoulis G. Large vessel occlusion strokes after the DIRECT-MT and SKIP trials: is the alteplase syringe half empty or half full? Stroke 2020;51:3182-6.

24 Goyal M, Menon BK, van Zwam WH, et al. Endovascular thrombectomy after large-vessel ischaemic stroke: a meta-analysis of individual patient data from five randomised trials. Lancet 2016;387:1723-31.

25 Goyal M, Almekhlafi M, Dippel DW, et al. Rapid alteplase administration improves functional outcomes in patients with stroke due to large vessel occlusions. Stroke 2019;50:645-51.

26 Katsanos $\mathrm{AH}$, Safouris A, Sarraj A, et al. Intravenous thrombolysis with tenecteplase in patients with large vessel occlusions: systematic review and meta-analysis. Stroke 2021;52:308-12.

27 Psychogios K, Palaiodimou L, Katsanos AH, et al. Real-world comparative safety and efficacy of tenecteplase versus alteplase in acute ischemic stroke patients with large vessel occlusion. Ther Adv Neurol Disord 2021;14:1756286420986727. 